\title{
PHOTODISSOCIATION REGIONS IN PLANETARY NEBULAE
}

\author{
V. ESCALANTE \\ Instituto de Astronomía, UNAM, Ap. Postal 70-264, México, DF 04510, México \\ and \\ A. GÓNGORA-T. \\ Instituto de Física, UNAM, Ap. Postal 20-364, México, DF 01000, México
}

\begin{abstract}
The photodissociation rate of $\mathrm{H}_{2}$ molecules by $\mathrm{UV}$ photons from PN central stars is generally several orders of magnitude larger than the rate produced by the average interstellar field (Sternberg 1988, Escalante et al. 1991). Thus, in neutral envelopes of PN's, $\mathrm{H}_{2}$ molecules are destroyed quickly, and a photodissociation region forms around ionization bounded nebulae. Observations of $\mathrm{H}_{2}$ in PN's reveal that not all the hydrogen is photodissociated, and it has been suggested that this is due to the existence of dense disks around the ionized region (Zuckerman and Gatley 1988).

In order to test different geometries and to examine the possibility that the atomic hydrogen observed in association with some PN's has been produced by radiation from the central star, we have used a photodissociation model in a gas with uniform temperature, and with formation rates of hydrogen molecules on grain surfaces typical of the interstellar medium. The model predicts the HI column density and mass, the $21 \mathrm{~cm}$ flux density and the brightness temperature profile. We have considered spherical envelopes with variable density $\left(n \propto r^{-2}\right)$ and disk structures like the ones observed in bipolar PN's with variable and constant density. We used expansion velocities measured by Taylor et al. (1990).

The results show that radiation of the central star can photodissociate the entire envelope in the cases with a spherical envelope and low column density $(N(\mathrm{H}) \lesssim$ $\left.5 \times 10^{20} \mathrm{~cm}^{-2}\right)$, and produce a region of extended low intensity, optically-thin $21 \mathrm{~cm}$ emission around the ionized zone. In the case of a molecular disk the central star may not photodissociate all the gas. The observed intensity of the $21 \mathrm{~cm}$ emission in NGC 6302, which is believed to have such a disk (Gómez et al. 1989), suggests that if the photodissociated region is formed in a disk-like envelope, the width of the disk must be considerably larger $(\gg 0.01 \mathrm{pc})$ than the typical dimensions of the ionized region. The mass of atomic $\mathrm{H}$ in spherical envelopes with variable density can be $\sim 0.1 M_{\odot}$, but it depends strongly on model parameters. For NGC 6302 we predict a mass of atomic $\mathrm{H}$ of $0.05 M_{\odot}$ in good agreement with the value obtained by Gómez et al. (1989).
\end{abstract}

\section{References}

Escalante, V., Sternberg, A., and Dalgarno, A., 1991, Ap. J., 375, 630.

Gómez, Y., Moran, J.M., Rodríguez, L.F., and Garay, G., 1989, Ap. J., 354, 862.

Sternberg, A., 1988, Ap. J., 332, 400.

Taylor, A.R., Gussie, G.T., and Pottasch, S.R., 1990, A p. J., 351, 515.

Zuckerman, B., and Gatley, I., 1988, Ap. J., 324, 501. 